TRANSACTIONS OF THE

AMERICAN MATHEMATICAL SOCIETY

Volume 350, Number 6, June 1998, Pages 2393-2407

S 0002-9947(98)01915-1

\title{
EIGENFUNCTIONS OF THE WEIL REPRESENTATION OF UNITARY GROUPS OF ONE VARIABLE
}

\author{
TONGHAI YANG
}

\begin{abstract}
In this paper, we construct explicit eigenfunctions of the local Weil representation on unitary groups of one variable in the $p$-adic case when $p$ is odd. The idea is to use the lattice model, and the results will be used to compute special values of certain Hecke $L$-functions in separate papers. We also recover Moen's results on when a local theta lifting from $U(1)$ to itself does not vanish.
\end{abstract}

\section{InTRODUCTION AND NotATION}

Let $E / F$ be a quadratic extension of local fields. If $(V,()$,$) is an Hermitian$ space over $E$, and $(W,\langle\rangle$,$) is a skew-Hermitian space over E$, the unitary groups $G=G(W)$ and $G^{\prime}=G(V)$ form a reductive dual pair in $\operatorname{Sp}(\mathbb{W})$, where $\mathbb{W}=V \otimes W$ has the symplectic form $\frac{1}{2} \operatorname{tr}_{E / F} \overline{(,)} \otimes\langle$,$\rangle over F$. According to a well-known result, this dual pair splits in the metaplectic cover of $\operatorname{Sp}(\mathbb{W})$, and thus has a Weil representation $\omega$. We consider the very special case in which $\operatorname{dim}_{E} V=\operatorname{dim}_{E} W=$ 1. In this case, $G \cong G^{\prime}=U(1)=E^{1}$ is compact and abelian, where $E^{1}$ is the kernel of the norm map $N: E^{*} \longrightarrow F^{*}$. So irreducible representations of $G$ are just characters, and the Weil representation has a direct sum decomposition:

$$
\omega=\bigoplus_{\eta} S(\eta)
$$

where the sum runs over characters of $G$, and $S(\eta)$ is the eigenspace of $(G, \omega)$ with eigencharacter $\eta$. Two questions arise naturally.

(1) What is $\operatorname{dim} S(\eta)$ ?

(2) When $S(\eta) \neq 0$, how do we find an explicit basis?

The first question is answered independently by Moen ([Moe], [Moe2]), Rogawski ([Ro]), and Harris, Kudla, and Sweet $([\mathrm{HKS}])$. One has $\operatorname{dim} S(\eta) \leq 1$. It is equal to 1 if and only if a certain dichotomy condition on root numbers is satisfied ([HKS, Corollary 8.5], [Ro, Theorem 1.1]). Moen gave another sufficient and necessary condition for $\operatorname{dim} S(\eta)=1$, case by case, which we will recover in this paper. The second question was answered in [Ya] in terms of classical Hermite functions when $F=\mathbb{R}$. In this paper, we will deal with the second question in the $p$-adic case with $p \neq 2$. Our motivation to construct such an explicit eigenfunction, in addition to

Received by the editors November 7, 1995 and, in revised form, July 3, 1996.

1991 Mathematics Subject Classification. Primary 11F27, $11 \mathrm{E} 45$.

Key words and phrases. Eigenfunction, lattice model, unitary group.

Partially supported by NSF grant DMS-9304580.

(C)1998 Americal Mathematical Society 
its own interest, is that they are needed to compute special central values of certain Hecke $L$-functions ([RVY], [Ya], [Ya2]).

Let $E / F$ be a quadratic extension of a $p$-adic local field with $p \neq 2$. We denote the nontrivial Galois automorphism of $E / F$ by $x \mapsto \bar{x}$. Let $E^{1}$ be the kernel of the norm map $N$. Choose and fix $\delta \in E^{*}-F^{*}$ such that $\bar{\delta}=-\delta$. So $\Delta=\delta^{2} \in F^{*}$. We assume for convenience (possible since $p \neq 2$ ) that $\delta$ is a uniformizer of $E$ when $E / F$ is ramified, and a unit when $E / F$ is unramified. Let $W=E$ with the skew-Hermitian form $\langle x, y\rangle=\delta x \bar{y}$, and let $G=G(W)=E^{1}$ be the isometry group acting on the right. Fix an $\alpha \in F^{*}$, and let $V_{\alpha}=E$ be the corresponding one-dimensional Hermitian space with the Hermitian form $(x, y)=\alpha \bar{x} y$ and $G_{\alpha}=G\left(V_{\alpha}\right)$ its isometry group, which acts on the left. Let $\mathbb{W}=V_{\alpha} \otimes_{E} W \cong E$ with the symplectic form

$$
\langle\langle x, y\rangle\rangle=\frac{\alpha}{2} \operatorname{tr}_{E / F} \delta x \bar{y}
$$

over $F$. There is a canonical map

$$
\imath_{\alpha}: \quad G \times G_{\alpha} \longrightarrow \mathrm{Sp}(\mathbb{W}) ; \quad(v \otimes w) \imath_{\alpha}\left(g, g_{1}\right)=g_{1}^{-1} v \otimes w g .
$$

This map makes $\left(G, G_{\alpha}\right)$ a reductive dual pair in $\operatorname{Sp}(\mathbb{W})=\operatorname{Sp}(1)$.

When $E / F$ is unramified, let $\pi=\pi_{F}=\pi_{E}$ be a uniformizer of $F$ and $E$. When $E / F$ is ramified, let $\pi_{E}=\delta$ and $\pi=\Delta$; they are uniformizers of of $E$ and $F$ respectively. Let

$$
G_{k}=\left\{g \in G: g \equiv 1 \bmod \pi_{E}^{k}\right\}
$$

and $G^{\prime}=G_{1} \times\{ \pm 1\}$.

Fix a nontrivial additive character $\psi$ of $F$ and let $\psi_{E}=\psi \circ \operatorname{tr}_{E / F}$ and $\psi^{\prime}=\frac{\delta \alpha}{4} \psi_{E}$. Then $\psi^{\prime}$ is an additive character of $E$ such that $\left.\psi^{\prime}\right|_{F}$ is trivial, and

$$
\psi^{\prime}(w \bar{z})=\psi\left(\frac{1}{2}\langle\langle w, z\rangle\rangle\right), \quad \text { for any } w, z \in \mathbb{W} .
$$

We write $n\left(\psi^{\prime}\right)$ for its conductor, i.e., the smallest integer $n$ such that $\left.\psi\right|_{\pi_{E}^{n} \mathcal{O}_{E}}$ is trivial.

Given a self-dual $\mathcal{O}_{F}$-lattice of $\mathbb{W}=E$ with respect to $\psi$ (section 2), one has a realization of the Weil representation $\omega_{\psi}$ of $\mathrm{Mp}(\mathbb{W})$ on the space (lattice model)

$$
S(L, \psi)=\left\{f \in S(E): f(z+l)=f(z) \psi^{\prime}(z \bar{l}) \text { for all } l \in L\right\}
$$

where $S(E)$ is the space of locally constant functions on $E$ with compact support. We refer the reader to $[\mathrm{MVW}]$ and its references for information on the lattice model.

Define $L_{n}=\pi_{E}^{n} \mathcal{O}_{E}$, and

$$
L= \begin{cases}\pi_{E}^{n} \mathcal{O}_{E} & \text { if } n\left(\psi^{\prime}\right)=2 n, \\ \pi^{n} \delta \mathcal{O}_{F} \oplus \pi^{n-1} \mathcal{O}_{F} & \text { if } n\left(\psi^{\prime}\right)=2 n-1 .\end{cases}
$$

Then $L$ is a self-dual lattice of $\mathbb{W}=E$ with respect to $\psi$. Given $w \in E$, we define a function $f_{w} \in S(L, \psi)$ via

$$
f_{w}(z)= \begin{cases}\psi^{\prime}(w \bar{z})=\psi\left(\frac{1}{2}\langle\langle w, z\rangle\rangle\right) & \text { if } z \in w+L_{n} \\ 0 & \text { otherwise. }\end{cases}
$$

In section 2, we will prove 
Theorem 0.1. Assume that $n\left(\psi^{\prime}\right)=2 n$ is even. Then

(1) The Weil representation $\omega_{\psi}$ of $G$ acts on $S(L, \psi)$ via right translation, and $G$ splits in $\mathrm{Mp}(\mathbb{W})$ trivially.

(2) The characteristic function $f_{0}$ of $L$ is the eigenfunction of $\left(G, \omega_{\psi}\right)$ with the trivial eigencharacter.

(3) Let $\eta$ be a nontrivial character of $G$ with (additive) conductor $2 k$ or $2 k-1>$ 0 . Then there is $b \in \mathcal{O}_{F}^{*}$ such that

$$
\eta(g)=\psi^{\prime}\left(-b\left(\pi_{E} \bar{\pi}_{E}\right)^{-k+n} g\right), \quad \text { for } g \in G_{k} .
$$

Moreover, $\eta$ occurs in the Weil representation $\omega_{\psi}$ of $G$ if and only if $b=a \bar{a} \in N E^{*}$.

(4) When $\eta$ occurs $\omega_{\psi}$,

$$
\phi_{\eta}=\sum_{g \in G / G_{k}} \eta(g) f_{a g \pi_{E}^{-k}}
$$

defines an eigenfunction (unique up to scalar) of $(G, \omega)$ with eigencharacter $\eta$.

In section 3, we deal with the case where $n\left(\psi^{\prime}\right)=2 n-1$ is odd. This can only happen when $E / F$ is unramified. Let $\pi$ be a uniformizer of $F$ and $E$. Let $G^{\prime}=G_{1} \times\{ \pm 1\}$. Let $\bar{F}$ be the residue field of $F$, and let $q$ be the order of $\bar{F}$.

Theorem 0.2 (Theorem 3.6). Assume that $n\left(\psi^{\prime}\right)=2 n-1$ is odd. Then $\omega_{\psi}(g)=$ $\lambda(g)^{-1} r(g)$ defines a Weil representation of $G$ on $S(L, \psi)$. Here

$$
\lambda(g)= \begin{cases}(x / \bar{F}) & \text { if } g \in G^{\prime}, \\ \gamma_{F}\left(\frac{\Delta \alpha}{2} y \psi\right) & \text { if } g \notin G^{\prime}\end{cases}
$$

where $(/ \bar{F})$ is the unique nontrivial quadratic character of $\bar{F}^{*}, \gamma_{F}$ is the local Weil index ([Wei], [Rao, appendix]), and

$$
r(g) f(z)= \begin{cases}f(z g) & \text { if } g \in G^{\prime}, \\ \frac{1}{\sqrt{q}} \sum_{a \in L / L_{n}} f(z g+a g) \psi^{\prime}(-z \bar{a}) & \text { if } g \notin G^{\prime}\end{cases}
$$

Theorem 0.3. Assume that $n\left(\psi^{\prime}\right)=2 n-1$ is odd. Let $\eta$ be a character of $G$ of conductor $n(\eta)>1$. Then

(1) $\eta$ occurs in $\omega_{\psi}$ if and only of $n(\eta)=2 k-1$ is odd $(k>1)$.

(2) When it occurs, there is $a \in \mathcal{O}_{E}^{*}-\left(\delta \mathcal{O}_{F}+\pi \mathcal{O}_{F}\right)$ such that

$$
\eta(g)=\psi^{\prime}\left(-a \bar{a} \pi^{-2 k+2 n} g\right), \quad \text { for } g \in G_{k} .
$$

Moreover,

$$
\phi_{\eta}=\sum_{g \in G / G_{k}} \eta(g)^{-1} \omega(g) f_{a \pi^{-k+n}}
$$

is an eigenfunction (unique up to scalar) of $G$ with eigencharacter $\eta$.

Let $\tilde{\eta}_{0}$ be the quadratic character of $E^{*}$ defined by $\tilde{\eta}_{0}(z)=(\pi, z \bar{z})_{F}$, where $(,)_{F}$ is the Hilbert symbol over $F$. Then $\left.\tilde{\eta}_{0}\right|_{F^{*}}$ is trivial, and there is a unique quadratic character $\eta_{0}$ of $G=E^{1}$ such that $\tilde{\eta}_{0}(z)=\eta_{0}\left(\frac{z}{\bar{z}}\right)$.

Theorem 0.4. Assume that $n\left(\psi^{\prime}\right)=2 n-1$ is odd. Then

(1) A character $\eta$ of $G$ of conductor $\leq 1$ occurs in $\omega_{\psi}$ if and only if $\eta \neq \eta_{0}$. 
When $\eta \neq \eta_{0}$, there is $w \in L_{n-1}$ such that

$$
\phi_{\eta}=\phi_{\eta, w}=\sum_{g \in G / G^{\prime}} \eta(g)^{-1} \omega(g)\left(f_{w}+\eta(-1)\left(\frac{-1}{\bar{F}}\right) f_{-w}\right) \neq 0
$$

is an eigenfunction (unique up to scalar) of $G$ with eigencharacter $\eta$. If $\eta(-1)=$ $(-1, \pi)_{F}$, then one can take $w=0$.

As we mentioned at the beginning, the conditions for $\eta$ to occur in $\omega_{\psi}$ given in this paper were already known to Moen ([Moe], [Moe2]).

\section{ACKNOWLEDGMENT}

The basic idea of this paper, using the lattice model instead of the Schrödinger model, was suggested to me by Jian-shu Li. I would like to thank him for this idea and for his advice. I also thank Jeffrey Adams and Stephen Kudla for their help during the preparation of this paper. Revision of this paper was done while the author is at the Institute for Advanced Study. I would like to thank IAS for its hospitality and its financial support. Finally, the author would like to thank the referee for his suggestions and comments on the early draft.

\section{Structure of $U(1)$}

The first two lemmas are well-known, and their proofs are straightforward.

Lemma 1.1. Assume that $E / F$ is unramified. Then

(a) $\left|G_{k} / G_{k+1}\right|=q$ for every $k \geq 1$.

(b) $\left|G / G_{1}\right|=q+1$. Moreover $G=\mu_{q+1} \times G_{1}$, where $\mu_{q+1}$ is the cyclic subgroup of $G$ generated by a primitive $(q+1)$ th root of 1 .

Lemma 1.2. Assume that $E / F$ is ramified. Then

(a) $G=\{ \pm 1\} \times G_{1}$.

(b) For any $k \geq 1, G_{2 k}=G_{2 k+1}$.

(c) For any $k \geq 1,\left|G_{2 k-1} / G_{2 k}\right|=q$.

Given a character $\eta$ of $G$ or $G_{k}$, the conductor of $\eta$ is defined to be the smallest integer such that $\left.\eta\right|_{G_{n}}=1$.

Proposition 1.3. Given an integer $n>1$, let $\psi_{n}$ be a fixed additive character of $E$ with conductor $\pi_{E}^{n} \mathcal{O}_{E}$ and $\left.\psi_{n}\right|_{F}=1$. Let $k$ be an integer with $\frac{n}{2} \leq k<n$.

(a) Given $a \in \mathcal{O}_{F}$, the map

$$
c_{a, k}: G_{k} \longrightarrow \mathbb{C}^{1}, \quad g \longmapsto \psi_{n}(a g)
$$

defines a character of $G_{k}$ of conductor $\leq n$. The equality holds if and only if $a \in \mathcal{O}_{F}^{*}$.

(b) Given a and $a^{\prime} \in \mathcal{O}_{F}^{*}$, one has

$$
c_{a, k}=c_{a^{\prime}, k} \Longleftrightarrow a \equiv a^{\prime} \bmod \pi_{E}^{n-k} \Longleftrightarrow a \equiv a^{\prime} \bmod U_{E}^{n-k} .
$$

Here $U_{E}^{k}=\left\{z \in \mathcal{O}_{E}^{*}: z \equiv 1 \bmod \pi_{E}^{k}\right\}$.

(c) Every character of $G_{k}$ of conductor $n$ has the form $c_{a, k}$ for some $a \in \mathcal{O}_{F}^{*}$.

Proof. (a) Let $g=1+z \in G_{k}$ with $z \in \pi_{E}^{k} \mathcal{O}_{E}$. Since $\left.\psi_{n}\right|_{F}=1$, one has

$$
c_{a, k}(g)=\psi_{n}(a z) \text {. }
$$


It is easy to see from (1.2) that $c_{a, k}$ is a character of $G_{k}$ of conductor less than or equal to $n$. Now assume $a \in \mathcal{O}_{F}^{*}$. Suppose $\left.c_{a, k}\right|_{G_{n-1}}=1$. For any $z \in \pi_{E}^{n-1} \mathcal{O}_{E}$, $\frac{1+z}{1+\bar{z}}=1+z-\bar{z}+z^{\prime} \in G_{n-1}$ for some $z^{\prime} \in \pi_{E}^{n} \mathcal{O}_{E}$. Thus

$$
1=c_{a, k}\left(\frac{1+z}{1+\bar{z}}\right)=\psi_{n}(a(z-\bar{z})) \text {. }
$$

On the other hand, $\psi_{n}(a(z+\bar{z}))=1$ since $a(z+\bar{z}) \in F$. So $\psi_{n}(2 a z)=1$ for any $z \in \pi_{E}^{n-1} \mathcal{O}_{E}$. Since $2 a \in \mathcal{O}_{F}^{*}$, one has then $\left.\psi_{n}\right|_{\pi_{E}^{n-1} \mathcal{O}_{E}}=1$, a contradiction. Therefore $c_{a, k}$ has conductor $n$ if $a \in \mathcal{O}_{F}^{*}$.

(b) It suffices to prove $c_{a, k}=c_{a^{\prime}, k} \Longleftrightarrow a \equiv a^{\prime} \bmod \pi_{E}^{n-k}$. Let $\pi_{E}$ be a uniformizer of $E$ with $\pi_{E}^{2} \in F$. Then we can write $a-a^{\prime}=\pi_{E}^{l} u$ with $u \in \mathcal{O}_{F}^{*}$ (since $\left.a-a^{\prime} \in F\right)$. So $c_{a-a^{\prime}, k}$ is $c_{u, k}$ with respect to $\pi_{E}^{l} \psi_{n}$, and thus has conductor $n-k-l$ by $(a)$. Therefore $c_{a-a^{\prime}, k}=1$ if and only if $l=n-k$; i.e., $a \equiv a^{\prime} \bmod \pi_{E}^{n-k}$.

(c) By $(a)$ and $(b)$, it suffices to show the following identity:

$$
\left|G_{k} / G_{n}\right|-\left|G_{k} / G_{n-1}\right|=\left|U_{F} / U_{F} \cap U_{E}^{n-k}\right| \text {. }
$$

When $E / F$ is unramified, the left hand side of (1.3) is equal to $q^{n-k}-q^{n-k-1}$ by Lemma 1.1. On the other hand, since $U_{F} \cap U_{E}^{n-k}=U_{F}^{n-k}$, the right hand side of (1.3) is equal to $\left|U_{F} / U_{F}^{n-k}\right|=q^{n-k}-q^{n-k-1}$. The identity (1.3) is true.

When $E / F$ is ramified, $n$ must be even since $\pi_{E}^{2 k} \mathcal{O}_{E} \subset F+\pi_{E}^{2 k+1} \mathcal{O}_{E}$. So the left hand side of $(1.3)$ is equal to $q^{\frac{n}{2}-\left[\frac{k}{2}\right]-1}(q-1)$ by Lemma 1.2. On the other hand, since $U_{F} \cap U_{E}^{n-k}=U_{F}^{\left[\frac{n-k+1}{2}\right]}=U_{F}^{\frac{n}{2}-\left[\frac{k}{2}\right]}$, the right hand side of (1.3) is also $q^{\frac{n}{2}-\left[\frac{k}{2}\right]-1}(q-1)$.

Corollary 1.4. In the notation of Proposition 1.3, every character $\chi$ of $G$ with conductor $n>1$ is an extension of a character $c_{a,\left[\frac{n+1}{2}\right]}$ of $G_{\left[\frac{n+1}{2}\right]}$ for some $a \in \mathcal{O}_{F}^{*}$. Moreover, a is uniquely determined modulo $U_{E}^{\left[\frac{n}{2}\right]}$ by $\chi$.

Remark 1.5. We remark that $a \in \mathcal{O}_{F}^{*}$ in Corollary 1.4 is determined by

$$
\chi\left(\frac{1+\pi_{E}^{k}}{1-\pi_{E}^{k}}\right)=\psi_{n}\left(2 a \pi_{E}^{k}\right), \quad k=\left[\frac{n+1}{2}\right] .
$$

\section{Eigenfunctions of $U(1)$}

Let $\psi$ be a fixed nontrivial additive character of $F$. Recall that $\psi^{\prime}=\frac{\delta \alpha}{4} \psi_{E}$ is trivial on $F$ and

$$
\psi^{\prime}(w \bar{z})=\psi\left(\frac{1}{2}\langle\langle w, z\rangle\rangle\right) \quad \text { for any } w, z \in E .
$$

Given an $\mathcal{O}_{F}$-lattice $L$ of $\mathbb{W}=E$, one defines its dual lattice with respect to $\psi$ via

$$
\begin{aligned}
L^{\perp} & =\{z \in \mathbb{W}: \psi(\langle\langle z, l\rangle\rangle)=1 \text { for } l \in L\} \\
& =\left\{z \in E: \psi^{\prime}(z \bar{l})=1 \text { for } l \in L\right\} .
\end{aligned}
$$

A lattice is self-dual (with respect to $\psi$ ) if $L^{\perp}=L$. Given a self-dual lattice $L$ of $\mathbb{W}$, one has a realization of the Weil representation $\omega_{\psi}$ of the metaplectic group $\mathrm{Mp}(\mathbb{W})$ on the space ([MVW, II8])

$$
S(L, \psi)=\left\{f \in S(E): f(z+l)=f(z) \psi^{\prime}(z \bar{l}) \text { for all } l \in L\right\},
$$


where $S(E)$ is the space of locally constant functions on $E$ with compact support. Let $K=K_{L}=\{g \in \mathrm{Sp}(\mathbb{W}): L g=L\}$. Then $K$ is a (maximal) compact subgroup of $\mathrm{Sp}(\mathbb{W})$ and splits trivially in $\mathrm{Mp}(\mathbb{W})$. The corresponding Weil representation of $K$ on $S(L, \psi)$ is given by right multiplication, i.e.

$$
\omega(k) f(z)=f(z k) \quad \text { for any } k \in K, \text { and } f \in S(L, \psi)
$$

Given an integer $n$, let $L_{n}=\pi_{E}^{n} \mathcal{O}_{E}$. Then $L_{n}$ are all the $\mathcal{O}_{E}$-lattices of $\mathbb{W}=E$. Straightforward calculation gives

Lemma 2.1. Let $L=\mathcal{O}_{F} e_{1} \oplus \mathcal{O}_{F} e_{2}$ be a $\mathcal{O}_{F}$-lattice of $\mathbb{W}$. Then $L^{\perp}=\pi^{k} L$ with $k=n(\psi)-\operatorname{ord}_{F}\left(\left\langle\left\langle e_{1}, e_{2}\right\rangle\right\rangle\right)$.

Lemma 2.2. The symplectic space $\mathbb{W}=E$ has a self-dual $\mathcal{O}_{E}$-lattice (with respect to $\psi$ ) if and only if $n\left(\psi^{\prime}\right)=2 n$ is even. In such a case, $L_{n}$ is the unique self-dual $\mathcal{O}_{E}$-lattice of $\mathbb{W}$ with $n=\frac{1}{2} n\left(\psi^{\prime}\right)$.

Lemma 2.3. If $E / F$ is ramified, then $n\left(\psi^{\prime}\right)$ is even.

Proof. Since $\operatorname{tr}_{E / F}\left(\pi_{E}^{2 n-1} \mathcal{O}_{E}\right)=\operatorname{tr}_{E / F}\left(\pi_{E}^{2 n} \mathcal{O}_{E}\right)=\pi_{F}^{n} \mathcal{O}_{F}, n\left(\psi_{E}\right)=2 n(\psi)-1$. Recall that $\pi_{E}=\delta$ is a uniformizer of $E$. Therefore

$$
n\left(\psi^{\prime}\right)=n\left(\psi_{E}\right)-\operatorname{ord}_{E}\left(\frac{\alpha \delta}{4}\right)=2 n(\psi)-2 \operatorname{ord}_{F}(\alpha)-2
$$

is even.

For the rest of this section, we assume that $n\left(\psi^{\prime}\right)=2 n$ is even and write $L=L_{n}$. We also fix a uniformizer $\pi_{E}$ of $E$. Since $G=G(W)$ preserves $L_{n}, G \subset K$ splits in $\mathrm{Mp}(\mathbb{W})$ trivially, and the corresponding Weil representation $\omega$ of $G$ on $S(L, \psi)$ is given via

$$
\omega(g) f(z)=f(z g) \text { for any } g \in G, f \in S(L, \psi), \text { and } z \in E .
$$

Given $w \in W$, we define

$$
f_{w}(z)= \begin{cases}\psi^{\prime}(w \bar{z})=\psi\left(\frac{1}{2}\langle\langle w, z\rangle\rangle\right) & \text { if } z \in w+L_{n}, \\ 0 & \text { otherwise. }\end{cases}
$$

Then $f_{w} \in S\left(L_{n}, \psi\right)$, and it is characterized by the conditions $\operatorname{supp}(f)=w+L_{n}$ and $f(w)=1$. Given $a \in \mathcal{O}_{E}$ and $k \geq 0$, let

$$
f_{a, k}=f_{a \pi_{E}^{-k+n}} .
$$

For every $g \in G$, one has

$$
\omega(g) f_{w}=f_{w g^{-1}}, \quad \omega(g) f_{a, k}=f_{a g^{-1}, k} .
$$

Lemma 2.4. (i) Given $w_{1}, w_{2} \in E$, the one-dimensional spaces $\mathbb{C} f_{w_{1}}=\mathbb{C} f_{w_{2}}$ if and only if $w_{1} \equiv w_{2} \bmod L_{n}$.

(ii) Given $a_{1}, a_{2} \in \mathcal{O}_{E}^{*}$ and $k_{1}, k_{2} \geq 0, \mathbb{C} f_{a_{1}, k_{1}}=\mathbb{C} f_{a_{2}, k_{2}}$ if and only if $k_{1}=k_{2}$ and $a_{1} \equiv a_{2} \bmod \pi_{E}^{k}$

Proof. Claim (ii) follows from $(i)$. If $\mathbb{C} f_{w_{1}}=\mathbb{C} f_{w_{2}}$, then $\operatorname{supp}\left(f_{w_{1}}\right)=\operatorname{supp}\left(f_{w_{2}}\right)$. So $w_{1} \equiv w_{2} \bmod L_{n}$. Conversely if $w_{1} \equiv w_{2} \bmod L_{n}$, then a simple calculation gives

$$
f_{w_{1}}=\psi^{\prime}\left(w_{1} \bar{w}_{2}\right) f_{w_{2}} .
$$

So $\mathbb{C} f_{w_{1}}=\mathbb{C} f_{w_{2}}$. 
For $k \geq 0$, define

$$
S_{k}(L, \psi)=\left\{f \in S(L, \psi): \operatorname{supp}(f) \subset \pi_{E}^{-k+n} \mathcal{O}_{E}^{*}+L\right\} .
$$

Then Lemma 2.4 and formula (2.7) imply the following:

Corollary 2.5. One has the G-invariant decompositions

$$
S(L, \psi)=\bigoplus_{k=0}^{\infty} S_{k}(L, \psi)
$$

and

$$
S_{k}(L, \psi)=\bigoplus_{a \in U_{E} / U_{E}^{k}} \mathbb{C} f_{a, k} .
$$

In particular, the characteristic function $f_{0}$ of $L_{n}$ is the eigenfunction of $G$ with the trivial eigencharacter.

Given an integer $k>0$, let

$$
\psi_{2 k}=-\left(\pi_{E} \bar{\pi}_{E}\right)^{-k+n} \psi^{\prime}=-\frac{\alpha \delta\left(\pi_{E} \bar{\pi}_{E}\right)^{-k+n}}{4} \psi_{E} .
$$

Then $\psi_{2 k}$ is a character of $E$ of conductor $\pi_{E}^{2 k} \mathcal{O}_{E}$ such that $\left.\psi_{2 k}\right|_{F}=1$, i.e. $\psi_{2 k}$ satisfies the condition in Proposition 1.3.

Lemma 2.6. Given $a \in \mathcal{O}_{E}^{*}$ and $k>0$, the function $f_{a, k}$ is an eigenfunction of $G_{k}$ with eigencharacter $c_{a \bar{a}, k}$. Here $c_{a \bar{a}, k}$ is the character of $G_{k}$ defined in Proposition 1.3 with respect to the character $\psi_{2 k}$ just defined.

Proof. It is a simple calculation. Indeed, given $g \in G_{k}$,

$$
\begin{aligned}
\omega(g) f_{a, k} & =f_{a g^{-1} \pi_{E}^{-k+n}} \\
& =f_{a, k} \psi^{\prime}\left(\pi_{E}^{-k+n} a g^{-1} \bar{\pi}_{E}^{-k+n} \bar{a}\right) \\
& =f_{a, k} \psi^{\prime}\left(-\left(\pi_{E} \bar{\pi}_{E}\right)^{-k+n} a \bar{a} g\right) \\
& =c_{a \bar{a}, k}(g) f_{a, k} .
\end{aligned}
$$

Theorem 2.7. Let the notation be as above.

(i) Let $\eta$ be a nontrivial character of $G$. If $\left.\eta\right|_{G_{k}}=c_{a \bar{a}, k}$ for some $k>0$ and $a \in \mathcal{O}_{E}^{*}$, then

$$
\phi_{\eta}=\sum_{g \in G / G_{k}} \eta(g) f_{a g, k}
$$

defines an eigenfunction of $G$ (via $\omega)$ with eigencharacter $\eta$. Otherwise, $\eta$ does not occur in the Weil representation $\omega$.

(ii) The Weil representation $(\omega, S(L, \psi))$ of $G$ has the $G$-invariant decomposition

$$
S(L, \psi)=\bigoplus_{k \geq 0} \bigoplus_{a \in U_{E} / G U_{E}^{k}} \bigoplus_{\left.\eta\right|_{G_{k}}=c_{a \bar{a}, k}} \mathbb{C} \phi_{\eta}
$$


Proof. By Lemma 2.6, $\eta(g) f_{a g, k}=\eta(g) \omega\left(g^{-1}\right) f_{a, k}$ depends only on the residue class $g G_{k}$, and thus $\phi_{\eta}$ is well-defined. Obviously $\omega(g) \phi_{\eta}=\eta(g) \phi_{\eta}$. One has also $\phi_{\eta} \neq 0$, since the $\operatorname{supp}\left(f_{a g, k}\right)$ are disjoint from each other when $g$ varies in $G / G_{k}$. This proves the first part of claim $(i)$. By Corollary 2.5,

$$
S(L, \psi)=\bigoplus S_{k}(L, \psi)
$$

and

$$
S_{k}(L, \psi)=\bigoplus_{a \in U_{E} / U_{E}^{k}} \mathbb{C} f_{a, k} .
$$

The group $G$ acts on $U_{E} / U_{E}^{k}$ by right multiplication. Given $a \in U_{E} / U_{E}^{k}$, its fixed subgroup $G_{a}=\left\{g \in G: a g \equiv a \bmod U_{E}^{k}\right\}=G_{k}$ is independent of $a$. So

$$
U_{E} / U_{E}^{k}=\bigcup_{a \in U_{E} / G U_{E}^{k}} \bigcup_{g \in G / G_{k}} a g U_{E}^{k},
$$

and

$$
S_{k}(L, \psi)=\bigoplus_{a \in U_{E} / G U_{E}^{k}} S_{a, k}(L, \psi)
$$

where

$$
S_{a, k}(L, \psi)=\bigoplus_{g \in G / G_{k}} \mathbb{C} f_{a g, k}
$$

is $G$-invariant. Note that $G_{k}$ acts on $S_{a, k}$ via $c_{a \bar{a}, k}$, and $\operatorname{dim} S_{a, k}(L, \psi)=\left|G / G_{k}\right|$. Therefore one has, by comparing dimensions, that

$$
S_{a, k}(L, \psi)=\bigoplus_{\left.\eta\right|_{G_{k}}=c_{a \bar{a}, k}} \mathbb{C} \phi_{\eta} .
$$

This proves $(i i)$. Now the other claim of $(i)$ is obvious.

We remark that $\phi_{\eta}$ depends on the choice of $a$. Indeed, if we denote $\phi_{\eta}$ by $\phi_{\eta, a}$, then $\phi_{\eta, a g}=\eta^{-1}(g) \phi_{\eta, a}$.

Corollary 2.8 (Moen). Let the notation be as above, and write $\phi_{\text {trivial }}=f_{0}$. Assume that $E / F$ is unramified and $n\left(\psi^{\prime}\right)=2 n$ is even. Then

$$
S(L, \psi)=\bigoplus_{\eta} \mathbb{C} \phi_{\eta}
$$

where the sum runs over all characters of $G$ of even conductor.

Proof. Since $c_{a \bar{a}, k}$ has even conductor $2 k$, any character of $G$ of odd conductor cannot appear in the decomposition (2.13) of $G$ by Theorem 2.7. Now assume that $\eta$ is a character of $G$ of even conductor $2 k$. By Corollary 1.4, $\left.\eta\right|_{G_{k}}=c_{b, k}$ for some $b \in \mathcal{O}_{F}^{*}$. Since $E / F$ is unramified, $b=a \bar{a}$ for some $a \in \mathcal{O}_{E}^{*}$. So $\eta$ appears in the decomposition (2.13) by Theorem 2.7 .

Corollary 2.9 (Moen). Assume that $E / F$ is ramified. Let $\eta$ be a character of $G$. Then

(i) The character $\eta$ has even conductor $2 k$ or 1 . When the conductor is even, $\left.\eta\right|_{G_{k}}=c_{b, k}$ for some $b \in \mathcal{O}_{F}^{*}$. 
(ii) When the conductor of $\eta$ is $2 k$, the local theta lifting $\theta_{G_{\alpha}}(\eta)$ does not vanish if and only if $b \in N_{E / F} \mathcal{O}_{E}^{*}$. The local theta lifting $\theta_{G_{\alpha}}(\eta)$ vanishes when the conductor of $\eta$ is 1 .

\section{Eigenfunctions of $U(1)$ : continued}

In this section, we assume that $n\left(\psi^{\prime}\right)=2 n-1$ is odd, where $\psi^{\prime}=\frac{\alpha \delta}{4} \psi_{E}$. By Lemma 2.3, $E=F(\delta)$ is unramified over $F$. Let $\pi=\pi_{E}=\pi_{F}$ be a uniformizer of both $E$ and $F$. Let $\bar{F}=\mathcal{O}_{F} / \pi \mathcal{O}_{F}$ be the residue field of $F$ and let $q=|\bar{F}|$ be the order of $\bar{F}$. One can easily verify the following:

Lemma 3.1. The lattice $L=\pi^{n} \delta \mathcal{O}_{F} \oplus \pi^{n-1} \mathcal{O}_{F}$ is a self-dual lattice of $\mathbb{W}=E$ with respect to $\psi$.

We will use this lattice throughout this section to realize the Weil representation $\omega_{\psi}$ of $\operatorname{Mp}(\mathbb{W})$ on $S(L, \psi)$. Let $G^{\prime}=\{g \in G: L g=L\}$. Then $G^{\prime}$ acts on $S(L, \psi)$ via right multiplication. It is not difficult to verify that

$$
G^{\prime}=\{g=x+y \delta \in G: y \equiv 0 \bmod \pi\}=\{ \pm 1\} \times G_{1} .
$$

Given $g \in G$ and $f \in S(L, \psi)$, we define

$$
r(g) f(z)= \begin{cases}f(z g) & \text { if } g \in G^{\prime}, \\ \frac{1}{\sqrt{q}} \sum_{a \in L / L_{n}} f(z g+a g) \psi^{\prime}(-z \bar{a}) & \text { if } g \notin G^{\prime},\end{cases}
$$

Then $r$ is a lifting of the projective Weil representation of $G$ on $S(L, \psi)$ ([MVW, II8]).

As in (2.5), given $w \in \mathbb{W}$, we define $f_{w} \in S(L, \psi)$ via

$$
f_{w}(z)= \begin{cases}\psi^{\prime}(w \bar{z}) & \text { if } z \in w+L, \\ 0 & \text { if } z \notin w+L .\end{cases}
$$

Then $r(g) f_{w}=f_{w g^{-1}}$ for $g \in G^{\prime}$, and

$$
f_{w}=\psi^{\prime}\left(w \bar{w}^{\prime}\right) f_{w^{\prime}} \quad \text { if } w \in w^{\prime}+L .
$$

Lemma 3.2. Let $g \in G-G^{\prime}$. Then

$$
r(g) f_{w}(z)= \begin{cases}0 & \text { if } z \notin w g^{-1}+L_{n-1}, \\ \frac{1}{\sqrt{q}} \psi^{\prime}(w \overline{z g+a g}) \psi^{\prime}(-z \bar{a}) & \text { if } z \in w g^{-1}+L_{n-1},\end{cases}
$$

where $a=a_{g}(w, z)$ is the unique element of $L$ (modulo $\left.L_{n}\right)$ such that $(z+a) g \in$ $w+L$. In particular, $\operatorname{supp}\left(r(g) f_{w}\right)=w g^{-1}+L_{n-1}$.

Proof. When $z \notin w g^{-1}+L_{n-1},(z+a) g \notin w+L_{n-1}$ for any $a \in L$. In particular, $(z+a) g \notin w+L$ for any $a \in L$. By definition, one has $r(g) f_{w}(z)=0$. Now assume $z \in w g^{-1}+L_{n-1}$, i.e. $w-z g \in L_{n-1}$. Therefore it is sufficient by definition to prove the following assertion: Given $w \in L_{n-1} / L$, there is a unique $a \in L / L_{n}$ such that $a g \in w+L$. Write

$$
w=\pi^{n-1} \delta u+L \in L_{n-1} / L, \quad a=\pi^{n-1} a^{\prime}+L_{n} \in L / L_{n}, \quad g=x+y \delta \in G-G^{\prime}
$$

with $u, a^{\prime}, x, y \in \mathcal{O}_{F}$. So $a g \in w+L$ simply means $a^{\prime} y \equiv u \bmod \pi$. Since $y \in \mathcal{O}_{F}^{*}$, $a^{\prime} \equiv y^{-1} u \bmod \pi$ exists and is unique.

Lemma 3.3. Formula (3.2) defines a unitary lifting of the projective Weil representation of $G$ on $S(L, \psi)$. 
Proof. It suffices to verify that $r(g)$ is a unitary operator on $S(L, \psi)$. Let $r^{\prime}$ be a unitary lifting of the projective Weil representation of $G$ on $S(L, \psi)$. Then there is a function $c(g): G \longrightarrow \mathbb{C}$ such that $r(g)=r^{\prime}(g) c(g)$. In particular,

$$
\langle r(g) f, r(g) f\rangle=|c(g)|^{2}\langle f, f\rangle
$$

for every $f \in S(L, \psi)$. Let $f=f_{0}=\operatorname{char}(L)$. When $g \in G^{\prime}, r(g) f_{0}=f_{0}$, and then $|c(g)|=1$ by (3.6). When $g \notin G^{\prime}$, one has by Lemma 3.4,

$$
\begin{aligned}
\left\langle r(g) f_{0}, r(g) f_{0}\right\rangle & =\frac{1}{q} \int_{L_{n-1}} d z \\
& =\frac{1}{q} \operatorname{meas}\left(L_{n-1}\right) \\
& =\operatorname{meas}(L)=\left\langle f_{0}, f_{0}\right\rangle .
\end{aligned}
$$

So $|c(g)|=1$. Therefore $r(g)$ is unitary.

By Lemma 3.3, $r$ defines a 2-cocycle $c: G \times G \longrightarrow \mathbb{C}^{1}$ via

$$
r\left(g_{1}\right) r\left(g_{2}\right)=c\left(g_{1}, g_{2}\right) r\left(g_{1} g_{2}\right) .
$$

Proposition 3.4. Given $g_{1}, g_{2} \in G$, write $g_{3}=g_{1} g_{2}$ and $g_{i}=x_{i}+y_{i} \delta$ with $x_{i}, y_{i} \in \mathcal{O}_{F}, i=1,2,3$. Then

$$
\begin{aligned}
c\left(g_{1}, g_{2}\right) & = \begin{cases}1 & \text { if } g_{1}, g_{2} \text { or } g_{3} \in G^{\prime}, \\
\gamma_{\bar{F}}\left(y_{1} y_{2} y_{3} \psi^{\prime \prime}\right) & \text { otherwise, }\end{cases} \\
& = \begin{cases}1 & \text { if } g_{1}, g_{2} \text { or } g_{3} \in G^{\prime}, \\
\gamma_{F}\left(\frac{\alpha \Delta}{2} y_{1} y_{2} y_{3} \psi\right) & \text { otherwise, }\end{cases}
\end{aligned}
$$

where $\psi^{\prime \prime}$ is the character of the residue field $\bar{F}$ induced by the character $\psi^{\prime \prime}=$ $\frac{\alpha \Delta}{2} \pi^{2 n-2} \psi: F \longrightarrow \mathbb{C}^{1}$, and $\gamma$ is the local Weil index (an 8th root of 1 ) defined by Weil ([Wei], see also [Rao]). Here we abuse the notation by identifying the residue character of $\psi^{\prime \prime}$ with $\psi^{\prime \prime}$ itself.

Proof. If $g_{1}, g_{2}$ or $g_{3} \in G^{\prime}$, say $g_{1} \in G^{\prime}$ but $g_{2}, g_{3} \notin G^{\prime}$, then $L g_{1}=L$. For any $f \in S(L, \psi)$,

$$
\begin{aligned}
r\left(g_{1}\right) r\left(g_{2}\right) f(z) & =r\left(g_{2}\right) f\left(z g_{1}\right) \\
& =\frac{1}{\sqrt{q}} \sum_{a \in L / L_{n}} f\left(z g_{1} g_{2}+a g_{2}\right) \psi\left(-\frac{1}{2}\left\langle\left\langle z g_{1}, a\right\rangle\right\rangle\right) \\
& =\frac{1}{\sqrt{q}} \sum_{a \in L / L_{n}} f\left(z g_{1} g_{2}+a g_{1} g_{2}\right) \psi\left(-\frac{1}{2}\left\langle\left\langle z g_{1}, a g_{1}\right\rangle\right\rangle\right) \\
& =r\left(g_{1} g_{2}\right) f(z) .
\end{aligned}
$$

So $c\left(g_{1}, g_{2}\right)=1$. Now assume $g_{i} \notin G^{\prime}$ for $i=1,2,3$. Then

$$
r\left(g_{1}\right) r\left(g_{2}\right) f_{0}(z)=\frac{1}{\sqrt{q}} \sum_{b \in L / L_{n}} r\left(g_{2}\right) f_{0}\left(z g_{1}+b g_{1}\right) \psi\left(-\frac{1}{2}\langle\langle z, b\rangle\rangle\right) .
$$


So

$$
\begin{aligned}
r\left(g_{1}\right) r\left(g_{2}\right) f_{0}(0) & =\frac{1}{\sqrt{q}} \sum_{b \in L / L_{n}} r\left(g_{2}\right) f_{0}\left(b g_{1}\right) \\
& =\frac{1}{\sqrt{q}} \sum_{b \in L / L_{n}} \psi^{\prime}\left(-b g_{1} \overline{a_{g_{2}}\left(0, b g_{1}\right)}\right) .
\end{aligned}
$$

On the other hand,

$$
r\left(g_{1}\right) r\left(g_{2}\right) f_{0}(0)=c\left(g_{1}, g_{2}\right) r\left(g_{1} g_{2}\right) f_{0}(0)=c\left(g_{1}, g_{2}\right) \frac{1}{\sqrt{q}} .
$$

So

$$
c\left(g_{1}, g_{2}\right)=\frac{1}{\sqrt{q}} \sum_{b \in L / L_{n}} \psi^{\prime}\left(-b g_{1} \overline{a_{g_{2}}\left(0, b g_{1}\right)}\right) .
$$

Write $b=\pi^{n-1} b^{\prime}$ and $a_{g_{2}}\left(0, b g_{1}\right)=\pi^{n-1} a^{\prime}$ with $a^{\prime}, b^{\prime} \in \mathcal{O}_{F}$. By Lemma 3.2, $a^{\prime}$ is given by the condition $\left(\pi^{n-1} b^{\prime} g_{1}+\pi^{n-1} a^{\prime}\right) g_{2} \in L$, i.e., $a^{\prime} \equiv-b^{\prime} y_{3} y_{2}^{-1} \bmod \pi$. Therefore (3.8) gives

$$
\begin{aligned}
c\left(g_{1}, g_{2}\right) & =\frac{1}{\sqrt{q}} \sum_{b^{\prime} \in \bar{F}} \psi^{\prime}\left(\pi^{n-1} b^{\prime} g_{1} \overline{\pi^{n-1} b^{\prime} y_{3} y_{2}^{-1}}\right) \\
& =\frac{1}{\sqrt{q}} \sum_{a \in \bar{F}} \psi^{\prime \prime}\left(a^{2} y_{1} y_{2} y_{3}\right) .
\end{aligned}
$$

Here we have used the facts that $\left.\psi^{\prime}\right|_{F}=1$ and that $\psi^{\prime \prime}=\left.\pi^{2 n-2} \delta \psi^{\prime}\right|_{F}=\frac{\alpha \Delta}{2} \pi^{2 n-2} \psi$ has conductor $\pi \mathcal{O}_{F}$ as a character of $F$ and can then be viewed as a nontrivial character of $\bar{F}$. Applying [Rao, Theorems A2 and A11], one has

$$
c\left(g_{1}, g_{2}\right)=\gamma_{\bar{F}}\left(y_{1} y_{2} y_{3} \psi^{\prime \prime}\right)=\gamma_{F}\left(\frac{\Delta \alpha}{2} y_{1} y_{2} y_{3} \psi\right) .
$$

Theorem 3.5. Given $g=x+y \delta \in G, x, y \in \mathcal{O}_{F}$, let

$$
\begin{aligned}
\lambda(g) & =\left\{\begin{array}{lr}
(x / \bar{F}) & \text { if } g \in G^{\prime}, \\
(y / \bar{F}) \gamma_{\bar{F}}\left(\psi^{\prime \prime}\right) & \text { if } g \notin G^{\prime},
\end{array}\right. \\
& = \begin{cases}(x / \bar{F}) & \text { if } g \in G^{\prime}, \\
\gamma_{F}\left(\frac{\Delta \alpha}{2} y \psi\right) & \text { if } g \notin G^{\prime},\end{cases}
\end{aligned}
$$

where $(/ \bar{F})$ is the unique nontrivial quadratic character of $\bar{F}^{*}$. Then

(a) One has

$$
c\left(g_{1}, g_{2}\right)=\lambda\left(g_{1}\right) \lambda\left(g_{2}\right) \lambda\left(g_{1} g_{2}\right)^{-1}
$$

So

$$
\tilde{\imath}_{\alpha}: G \longrightarrow \mathrm{Mp}(\mathbb{W})=\mathrm{Sp}(\mathbb{W}) \times \mathbb{C}^{1}, \quad g \mapsto\left(\imath_{\alpha}(g), \lambda^{-1}(g)\right)
$$

gives a splitting of $G$ in $\mathrm{Mp}(\mathbb{W})$, where $\mathrm{Mp}(\mathbb{W})=\mathrm{Sp}(\mathbb{W}) \times \mathbb{C}^{1}$ is a lattice model realization of $\mathrm{Mp}(\mathbb{W})$ corresponding to the lattice $L$.

(b) The formula $\omega(g)=\lambda^{-1}(g) r(g)$ gives a Weil representation of $G$ on $S(L, \psi)$. 
Proof. Claim $(b)$ follows from $(a)$. By [Rao, A11], $\gamma_{F}\left(\frac{\Delta \alpha}{2} y \psi\right)=\gamma_{\bar{F}}\left(y \psi^{\prime \prime}\right)=$ $(y / \bar{F}) \gamma_{\bar{F}}\left(\psi^{\prime \prime}\right)$, so the two formulas for $\lambda(g)$ are the same. Write $g_{3}=g_{1} g_{2}$ and $g_{i}=x_{i}+y_{i} \delta$.

If $g_{i} \notin G^{\prime}$, for $i=1,2,3$, one has, by [Rao, A9],

$$
\lambda\left(g_{1}\right) \lambda\left(g_{2}\right) \lambda\left(g_{3}\right)^{-1}=\left(\frac{y_{1} y_{2} y_{3}}{\bar{F}}\right) \gamma_{\bar{F}}\left(\psi^{\prime \prime}\right)=\gamma_{\bar{F}}\left(y_{1} y_{2} y_{3} \psi^{\prime \prime}\right)=c\left(g_{1}, g_{2}\right) .
$$

If exactly one of the $g_{i}$ is in $G^{\prime}$, say $g_{1} \in G^{\prime}$, then $y_{1} \equiv 0 \bmod \pi$ and $y_{3}=x_{1} y_{2}+$ $x_{2} y_{1} \equiv x_{1} y_{2} \bmod \pi$. By [Rao, A9],

$$
\lambda\left(g_{1}\right) \lambda\left(g_{2}\right) \lambda\left(g_{3}\right)^{-1}=\left(\frac{x_{1}}{\bar{F}}\right)\left(\frac{y_{2}}{\bar{F}}\right) \gamma_{\bar{F}}\left(\psi^{\prime \prime}\right)\left(\frac{y_{3}}{\bar{F}}\right) \gamma_{\bar{F}}\left(\psi^{\prime \prime}\right)^{-1}=1=c\left(g_{1}, g_{2}\right) .
$$

Finally, if $g_{i} \in G^{\prime}$ for all $i=1,2,3$, then $y_{i} \equiv 0 \bmod \pi$ and so $x_{3} \equiv x_{1} x_{2} \bmod \pi$. One has also

$$
\lambda\left(g_{1}\right) \lambda\left(g_{2}\right) \lambda\left(g_{3}\right)^{-1}=1=c\left(g_{1}, g_{2}\right) .
$$

Remark 3.6. As Jeffrey Adams and Stephen Kudla pointed out, the Weil representation $\omega$ of $G$ constructed in (2.4) and Theorem 3.5 gives rise to a $\mu_{4}$-splitting of $G$ in $\mathrm{Mp}(\mathbb{W})$, i.e. under some realization $\mathrm{Mp}(\mathbb{W})=\mathrm{Sp}(\mathbb{W}) \times \mathbb{C}^{1}$, one has a splitting

$$
\tilde{\imath}_{\alpha}: G \longrightarrow \mathrm{Mp}(\mathbb{W}), \quad g \mapsto\left(\imath_{\alpha}(g), \lambda(g)\right)
$$

such that $\lambda(g) \in \mu_{4}=$ the group of 4 th roots of 1 . Recently, Adams has found a $\mu_{2}$-splitting of $G$ in $\operatorname{Mp}(\mathbf{W})$. Note that in the case $F=\mathbb{R}$, there is no $\mu_{n}$-splitting of $G$ in $\mathrm{Mp}(\mathbb{W})$ for any integer $n \geq 1$.

Lemma 3.7. The Weil representation $\omega$ of $G$ has the decomposition:

$$
S(L, \psi)=\bigoplus_{k=1}^{\infty} S_{k}(L, \psi)
$$

where

$$
\begin{aligned}
& S_{1}(L, \psi)=\left\{f \in S(L, \psi): \operatorname{supp}(f) \subset L_{n-1}\right\}, \\
& S_{k}(L, \psi)=\left\{f \in S(L, \psi): \operatorname{supp}(f) \subset L_{n-k}-L_{n-k+1}\right\} \quad \text { for } k>1 .
\end{aligned}
$$

As in section 2,

$$
\psi_{2 k-1}=-\pi^{-2 k+2 n} \psi^{\prime}=-\frac{\alpha \delta}{4} \pi^{-2 k+2 n} \psi_{E}
$$

defines a character of $E$ with conductor $\pi^{2 k-1} \mathcal{O}_{E}$, and $\left.\psi_{2 k-1}\right|_{F}=1$. So, given $a \in \mathcal{O}_{F}^{*}$, one has a character $c_{a, k}$ of $G_{k}$ of conductor $2 k-1$, defined by (Proposition $1.3)$

$$
c_{a, k}(g)=\psi_{2 k-1}(a g)=\psi^{\prime}\left(-\pi^{-2 k+2 n} a g\right) .
$$

Similarly to Lemma 2.6, one has

Lemma 3.8. Let $k>1$ and $a \in \mathcal{O}_{E}^{*}$. Then $f_{a, k}=f_{a \pi^{-k+n}}$ is an eigenfunction of $G_{k}$ with eigencharacter $c_{a \bar{a}, k}$. Moreover, $c_{a \bar{a}, k}=c_{a^{\prime} \bar{a}^{\prime}, k}$ if and only if $a \in a^{\prime} G U_{E}^{k-1}$. 
Proof of Theorem 0.3. First assume that $n(\eta)=2 k-1>1$ is odd. By Proposition 1.3 , there is $a \in \mathcal{O}_{E}^{*}$ such that $\left.\eta\right|_{G_{k}}=c_{a \bar{a}, k}$. By Lemma 3.8, one may assume that $a \notin \delta \mathcal{O}_{F}+\pi \mathcal{O}_{F}$. Applying Lemma 3.8 again, one has that

$$
\phi_{\eta}=\phi_{\eta, a, k}=\sum_{g \in G / G_{k}} \eta(g)^{-1} \omega(g) f_{a, k}
$$

is an eigenfunction of $\left(G, \omega_{\psi}\right)$, with eigencharacter $\eta$ if it is nonzero. Here $f_{a, k}=$ $f_{a \pi^{-k+n}}$. To check that $\phi_{\eta} \neq 0$, it suffice to verify that the supports of $\omega(g) f_{a, k}$ and $f_{a, k}$ are disjoint unless $g \in G_{k}$. In fact,

$$
\begin{aligned}
\operatorname{supp}\left(\omega(g) f_{a, k}\right) & \subset \pi^{-k+n} a g^{-1}+L_{n-1}, \\
\operatorname{supp}\left(f_{a, k}\right) & =\pi^{-k+n} a+L \subset \pi^{-k+n} a+L_{n-1} .
\end{aligned}
$$

If they are not disjoint, then $\pi^{-k+n} a\left(1-g^{-1}\right) \in L_{n-1}$, and so $g \in G_{k-1}$. Write $g^{-1}=\frac{1+\pi^{k-1} z}{1+\pi^{k-1} \bar{z}} \in G$ for some $z \in \mathcal{O}_{E}$; then

$$
g^{-1} \equiv 1+\pi^{k-1}(z-\bar{z}) \bmod \pi^{k} \equiv 1+\pi^{k-1} x \delta \bmod \pi^{k}
$$

for some $x \in \mathcal{O}_{F}$. Write also $a=b \delta+c$, with $c \in \mathcal{O}_{F}^{*}$ by assumption. Now $g \in G_{k-1}$ implies that $\omega(g) f_{a, k}=f_{a g^{-1}, k}$ has support $\pi^{-k+n} a g^{-1}+L$. So we should have $\pi^{-k+n} a\left(1-g^{-1}\right) \in L$; i.e., $(b \delta+c) \delta x \in \pi \delta \mathcal{O}_{F}+\mathcal{O}_{F}$. So $x \in \pi \mathcal{O}_{F}$ and $g \in G_{k}$. Now it remain to prove that if $\eta$ occurs in $\omega_{\psi}$ and $n(\eta)>1$, then $n(\eta)$ is odd. Obviously,

$$
S_{k}(L, \psi) \supset \bigoplus_{a \in U_{E} / G U_{E}^{k-1}} \bigoplus_{\left.\eta\right|_{G_{k}}=c_{a \bar{a}, k}} \mathbb{C} \phi_{\eta} .
$$

On the other hand, $S_{k}(L, \psi)=\bigoplus_{w \in \pi^{-k+n} \mathcal{O}_{E}^{*} / L} \mathbb{C} f_{w}$ implies

$$
\operatorname{dim} S_{k}(L, \psi)=\left|\pi^{-k+n} \mathcal{O}_{E}^{*} / L\right|=q^{2 k-3}\left(q^{2}-1\right) .
$$

By Corollary 1.4, $\left\{\eta:\left.\eta\right|_{G_{k}}=c_{a \bar{a}, k}, a \in U_{E} / G U_{E}^{(k-1)}\right\}$ counts all characters of $G$ of conductor $2 k-1$. So the right hand side of (3.13) has dimension

$$
\left|G / G_{2 k-1}\right|-\left|G / G_{2 k-2}\right|=(q+1) q^{2 k-2}-(q+1) q^{2 k-3}=q^{2 k-3}\left(q^{2}-1\right) .
$$

This proves that

$$
S_{k}(L, \psi)=\bigoplus_{a \in U_{E} /\left.G U_{E}^{(k-1)} \eta\right|_{G_{k}}=c_{a \bar{a}, k}} \mathbb{C} \phi_{\eta} .
$$

Recall that $n(\eta)=2 k-1$ if $\left.\eta\right|_{G_{k}}=c_{a \bar{a}, k}$ for some $a \in \mathcal{O}_{E}^{*}$. This completes the proof of Theorem 0.3 .

Proof of Theorem 0.4. First note that $S_{1}(L, \psi)$ has an orthogonal basis $\left\{f_{w}: w \in\right.$ $\left.L_{n-1} / L\right\}$ :

$$
\left\langle f_{w}, f_{w^{\prime}}\right\rangle= \begin{cases}0 & \text { if } w \notin w^{\prime}+L, \\ \operatorname{meas}(L) & \text { if } w=w^{\prime}\end{cases}
$$

For each $w \in L_{n-1} / L$, choose and fix a representative $w=\pi^{n-1} \delta u, u \in \mathcal{O}_{F}$. Since $\left|S_{1}(L, \psi)\right|=q$ and $\left|G / G_{1}\right|=q+1$, the multiplicity one theorem implies that there is a unique character $\eta_{0}$ of $G / G_{1}$ such that

$$
S_{1}(L, \psi)=\bigoplus_{\eta \neq \eta_{0}} \mathbb{C} \phi_{\eta},
$$


where the sum runs over all characters of $G / G_{1}$ with $\eta \neq \eta_{0}$. We need to show that $\eta_{0}$ is the one given in the introduction. Let $r$ be the regular representation of $G / G_{1}$, also viewed as a representation of $G$. Let $\omega_{1}=\left.\omega\right|_{S_{1}(L, \psi)}$. By (3.19), one has $r=\omega_{1} \oplus \eta_{0}$, i.e., $\operatorname{char}(r)=\operatorname{char}\left(\omega_{1}\right)+\eta_{0}$, where $\operatorname{char}(r)$ is the character of $r$. Therefore for any $g \notin G_{1}$, one has

$$
\eta_{0}(g)=-\operatorname{char}\left(\omega_{1}\right)(g) .
$$

By (3.18), one has

$$
\operatorname{char}\left(\omega_{1}\right)(g)=\frac{1}{\operatorname{meas}(L)} \sum_{w \in L_{n-1} / L}\left\langle\omega(g) f_{w}, f_{w}\right\rangle .
$$

When $g \in G^{\prime}-G_{1}, \omega(g) f_{w}=(-1 / \bar{F}) f_{-w}$. So $(3.18)$ implies $\eta_{0}(g)=(-\Delta / \bar{F})$. We may now assume $g=x+y \delta \notin G^{\prime}, y \in \mathcal{O}_{F}^{*}$. Then

$$
\begin{aligned}
\left\langle\omega(g) f_{w}, f_{w}\right\rangle & =\int_{w+L} \omega(g) f_{w}(z) \overline{f_{w}(z)} d z \\
& =\lambda^{-1}(g) \int_{L} r(g) f_{w}(w+z) \overline{\psi^{\prime}(w \bar{z})} d z .
\end{aligned}
$$

Write $w=\pi^{n-1} \delta u, z=\pi^{n} \delta b+\pi^{n-1} c$. Let $a=-c+u y^{-1}(1-x)$. Then we have $\left(w+z+\pi^{n-1} a\right) g \in w+L$. By Lemma 3.2,

$$
\begin{aligned}
r(g) f_{w}(w+z) & =\frac{1}{\sqrt{q}} \psi^{\prime}\left(w \overline{\left(w+z+\pi^{n-1} a\right) g}\right) \psi^{\prime}\left(-(w+z) \pi^{n-1} a\right) \\
& =\frac{1}{\sqrt{q}} \psi^{\prime}\left(\pi^{2 n-2} \delta u^{2} y^{-1}(x-1)\right) \psi^{\prime}\left(\pi^{2 n-2} \delta u c\right) .
\end{aligned}
$$

So

$$
\begin{aligned}
\left\langle\omega(g) f_{w}, f_{w}\right\rangle & =\frac{1}{\sqrt{q} \lambda(g)} \int_{L} \psi^{\prime}\left(2 \pi^{2 n-2} \delta u^{2} y^{-1}(x-1)\right) d z \\
& =\frac{1}{\sqrt{q} \lambda(g)} \psi^{\prime \prime}\left(\frac{2(x-1)}{y} u^{2}\right) \operatorname{meas}(L) .
\end{aligned}
$$

Therefore

$$
\begin{aligned}
\operatorname{char}\left(\omega_{1}\right)(g) & =\frac{1}{\sqrt{q} \lambda(g)} \sum_{u \in \bar{F}} \psi^{\prime \prime}\left(\frac{2(x-1)}{y} u^{2}\right) \\
& =\lambda^{-1}(g) \gamma_{\bar{F}}\left(2 y(x-1) \psi^{\prime \prime}\right) \\
& =\left(\frac{2(x-1)}{\bar{F}}\right) .
\end{aligned}
$$

So

$$
\eta_{0}(g)=-\left(\frac{2(x-1)}{\bar{F}}\right)=\left(\frac{2 \Delta(x-1)}{\bar{F}}\right) .
$$

In summary, one has always

$$
\eta_{0}(g)=\left(\frac{2 \Delta(x-1)}{\bar{F}}\right) .
$$

It is easy to see from (3.22) that $\eta_{0}$ is indeed the character $\eta_{0}$ defined in the introduction. This proves (1). 
To prove (2), first notice that

$$
\phi_{\eta, w}=\sum_{g \in G / G_{1}} \eta(g)^{-1} \omega(g) f_{w}
$$

is well-defined and is an eigenfunction of $(G, \omega)$ with eigencharacter $\eta$ if it is nonzero. If it is zero for every $w \in L_{n-1}$, then

$$
\sum_{g \in G / G_{1}} \eta(g)^{-1} \omega(g) f=0
$$

for every $f \in S_{1}(L, \psi)$. On the other hand, let $\phi \in S_{1}(L, \psi)$ be an eigenfunction of $G$ with eigencharacter $\eta$ (we have just proved that it exists), and let $f=\frac{1}{q+1} \phi$. Then

$$
\phi=\sum_{g \in G / G_{1}} \eta(g)^{-1} \omega(g) f=0,
$$

a contradiction. So there is $w \in L_{n-1}$ such that $\phi_{\eta, w} \neq 0$. When $\eta(-1)=(-1 / \bar{F})$,

$$
\phi_{\eta, 0}(0)=2+2 \frac{1}{\sqrt{q}} \sum_{g \in G / G^{\prime}, g \neq 1}(\eta(g) \lambda(g))^{-1} .
$$

Since $\eta(g) \lambda(g) \in \mathbb{Q}\left(\zeta_{2(q+1)}\right)$ and $\sqrt{q} \notin \mathbb{Q}\left(\zeta_{2(q+1)}\right), \phi_{\eta, 0}(0) \neq 0$. Here $\zeta_{2(q+1)}$ is a primitive $2(q+1)$ th root of 1 .

\section{REFERENCES}

[HKS] M. Harris, S. Kudla and W. Sweet, Theta dichotomy for unitary groups, J. Amer. Math. Soc. 9 (1996), 941-1004. MR 96m:11041

[Ka] S. Kudla, Splitting metaplectic covers of dual reductive pairs, Israel J. Math. 87 (1994), 361-401. MR 95h:22019

[Li] Jian-shu Li, Theta series, Lecture notes at University of Maryland, College Park, 1992.

[Moe] C. Moen, The dual pair $(U(3), U(1))$ over a p-adic field, Pacific J. Math. 127 (1987), 141-154. MR 88e:22035

[Moe2] The dual pair $(U(1), U(1))$ over a p-adic field, Pacific J. Math. 158 (1993), 365-386. MR 94a:22036

[MVW] C. Moeglin, M.-F.Vigneras, J.-L. Waldspurger, Correspondances de Howe sur un corps p-adiques, Lecture Notes in Math., vol. 1291, Springer-Verlag, New York, 1987. MR 91f: 11040

[Rao] R. Ranga Rao, On some explicit formulas in the theory of Weil representations, Pacific J. Math 157 (1993), 335-371. MR 94a:22037

[Ro] J. D. Rogawski, The multiplicity formula for A-packets, The Zeta Function of Picard Modular Surfaces (R. P. Langlands and D. Pramakrishnan, editors), Centre de Recherches Math., Univ. Montréal, Montréal, 1992, pp. 395-419. MR 93f: 11042

[RVY] F. Rodriguez Villegas and T.H. Yang, Special values of L-functions of powers of canonical Hecke characters at the central point, to appear in Duke Math. J.

[Was] L. Washington, Introduction to cyclotomic fields, GTM 83, Springer-Verlag, 1982. MR 85g:11001

[Wei] A. Weil, Sur la formule de Siegel dans la théorie des groupes classiques, Acta Math. 123 (1965), 1-87. MR 36:6421

[Ya] Tonghai Yang, Theta liftings and Hecke L-functions, J. Reine Angew. Math. 485 (1997), 25-53. CMP 97:10

[Ya2] Nonvanishing of central Hecke L-value and rank of associated elliptic curves, to appear in Compositio Math.

Department of Mathematics, University of Michigan, Ann Arbor, Michigan 48109

E-mail address: thyang@math.lsa.umich.edu 